\title{
Carcinosarcoma of the Stomach: A Case Report
}

\author{
Kyeong Woon Choi, Woo Yong Lee, Seong Woo Hong, Yeo Goo Chang, Byungmo Lee, and Hye Kyung Lee ${ }^{1}$ \\ Department of Surgery, ${ }^{1}$ Department of Pathology, Inje University Paik Hospital, Inje University College of Medicine, Seoul, Korea
}

Carcinosarcoma is a rare malignant, biphasic tumor comprised of carcinoma and sarcoma components. In the gastrointestinal tract, carcinosarcoma is most frequently seen in the esophagus and rarely in the stomach. We report a 51-year-old female patient with 2-monthhistory of epigastric pain and dyspepsia. Endoscopic finding revealed a huge ulcerative lesion that infiltrated from the antrum to the mid-body. An endoscopically taken biopsy revealed poorly differentiated malignant round cell neoplasm. After the palliative subtotal gastrectomy, immunohistochemical studies showed two positive reactions for the epithelial marker and mesenchymal marker. Based on the above findings, the patient was diagnosed with gastric carcinosarcoma. The immunohistochemical analysis is a critical method in making an accurate diagnosis of carcinosarcoma.

Key Words: Carcinosarcoma; Stomach; Immunohistochemistry

\section{Introduction}

Carcinosarcoma is a malignant tumor consisting of both epithelial and mesenchymal components that rarely occurs in stomach. It typically occurs in uterus, breast, thyroid, lung, and upper gastrointestinal system. ${ }^{1}$ Endoscopic characteristics include excessive thickening of the gastric wall and huge ulcerative tumor, which are easily mistaken for Borrman type III of gastric adenocarcinoma. ${ }^{2,3}$ Histological identification of the coexistence of carcinomatous and sarcomatous components of the tumor as well as the immunohistochemical (IHC) analysis is critical for diagnosis. We report a case of a 51-year-old woman with gastric carcinosarcoma along with its clinical, macroscopic and histopathologic features.

Correspondence to: Woo Yong Lee

Department of Surgery, Inje University Paik Hospital, Inje University College of Medicine, 9, Mareunnae-ro, Jung-gu, Seoul 100-032, Korea Tel: +82-2-2270-0247, Fax: +82-2-2270-0250

E-mail: yongaaa5972@naver.com

Received August 29, 2012

Revised November 21, 2012

Accepted December 1, 2012

\section{Case Report}

The patient was a 51-year-old woman with 2-month-history of epigastric pain, dysphagia, anorexia, weight loss (4 kilos over 2 months, 7.2\%), and intermittent bloody vomit. At the time of admission, patient had an acute ill-looking appearance. General examination revealed marked pallor and tenderness in the epigastric region. Most of routine laboratory parameters were found to be in normal range except for the markers of hypochromic anemia: hemoglobin $6.1 \mathrm{~g} / \mathrm{dl}$, hematocrit $19 \%$, mean corpuscular volume $85.5 \mathrm{fL}$, iron blood level $9 \mu \mathrm{g} / \mathrm{dl}$, iron-binding capacity $222 \mu \mathrm{g} / \mathrm{dl}$, and erythrocyte sedimentation rate $120 \mathrm{~mm} / \mathrm{h}$. The levels of tumor markers, carcinoembryonic antigen (CEA) and carbohydrate antigen (CA 19-9) were $1.1 \mathrm{ng} / \mathrm{ml}$ and $9.7 \mathrm{ng} / \mathrm{ml}$, respectively (normal CEA $<5$, CA 19-9<37).

Endoscopic examination revealed a huge ulcerative lesion that infiltrated from the antrum to the mid-body with its base covered with old blood clot. A preoperative endoscopic biopsy revealed the poorly differentiated, invasive malignant round cell neoplasm (Fig. 1). Howerever, it did not determined specific histologic type due to severe tissue necrosis and hemorrhage. However, no definitive diagnosis could be reached using conventional $\mathrm{H} \& \mathrm{E}$ staining alone. 
Choi KW, et al

For more accurate diagnosis, additional IHC stains are required. Computed tomography detected localized wall thickening in gastric body with a few enlarged perigastric lymph nodes and there was no evidence of peritoneal seeding (Fig. 2).

The patient subsequently underwent palliative subtotal gastrectomy due to seeding over peritoneum, mesentery, and transverse colon and bleeding in the tumor. Macroscopically, a specimen of $12.0 \times 10.1 \mathrm{~cm}$ sized exophytic mass (Borrmann type II) was found which involved the antrum and mid-body along lesser curvature. Lesions of necrosis, hemorrhage, and focal penetrated perforation were observed in the tumor (Fig. 3).

According to pathology report, 3 metastatic lymph nodes were

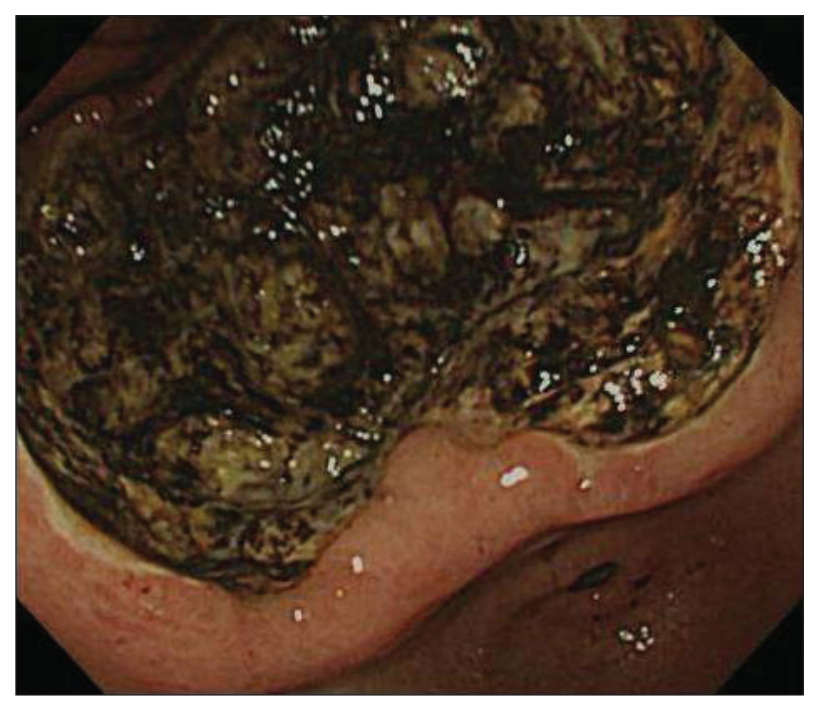

Fig. 1. Gastrofiberscopic examination revealed a huge ulcerofungating tumor with an extensive central ulceration and a peripheral fungating mound.

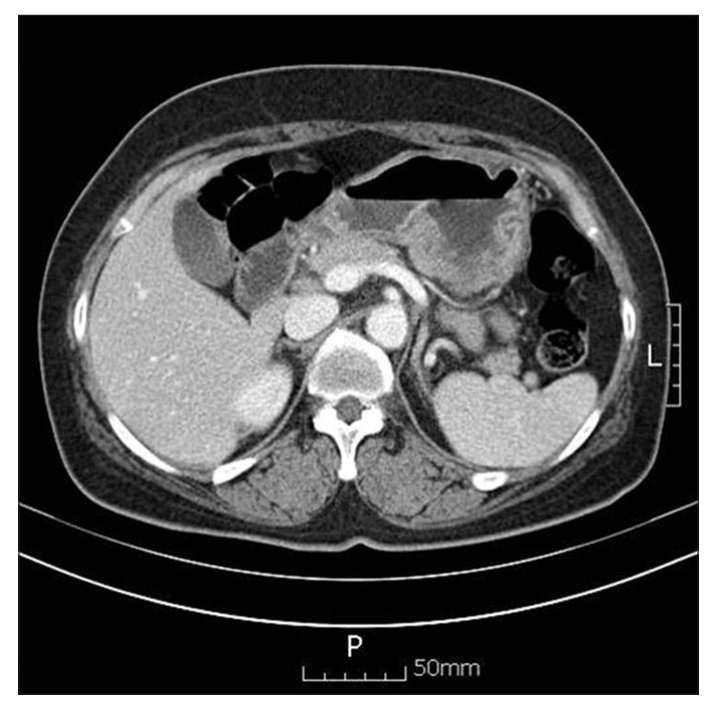

Fig. 2. Computed tomography showed diffuse wall thickening in gastric body, with a few enlarged lymph nodes. identified among 35 regional perigastric lymph nodes along with peritoneal seeding. These metastatic lymph nodes harbored carcinoma component (according to 6th international Union Against Cancer TNM staging system: T4, N1, M1).

The tumor showed mixed undifferentiated carcinoma and sarcoma in conventional H\&E staining (Fig. 4). However, for no definite diagnosis could be made using conventional H\&E staining alone, IHC analysis was performed. The tumor was compatible with undifferentiated carcinosarcoma which was positive for epithelial marker (cytokeratin, epithelial membrane antigen [EMA], BerEP4) and mesenchymal marker (vimentin, CD117, S100protein, CD68, smooth muscle actin, desmin) while partially being unresponsive for mesothelial marker (calretinin) (Fig. 5). Carcinoma component was not involved in definite adenocarcinomatous differentiation but in neuroendocrine marker (CD56, neuron specific

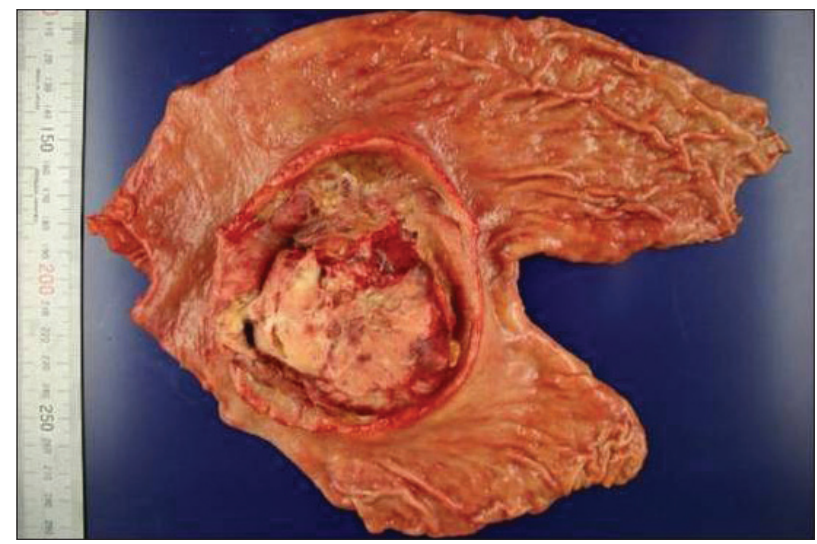

Fig. 3. Macroscopic finding is an oval, relatively well-defined, ulcerofungating tumor with an extensive, excavating central ulceration and a peripheral, fungating margin.

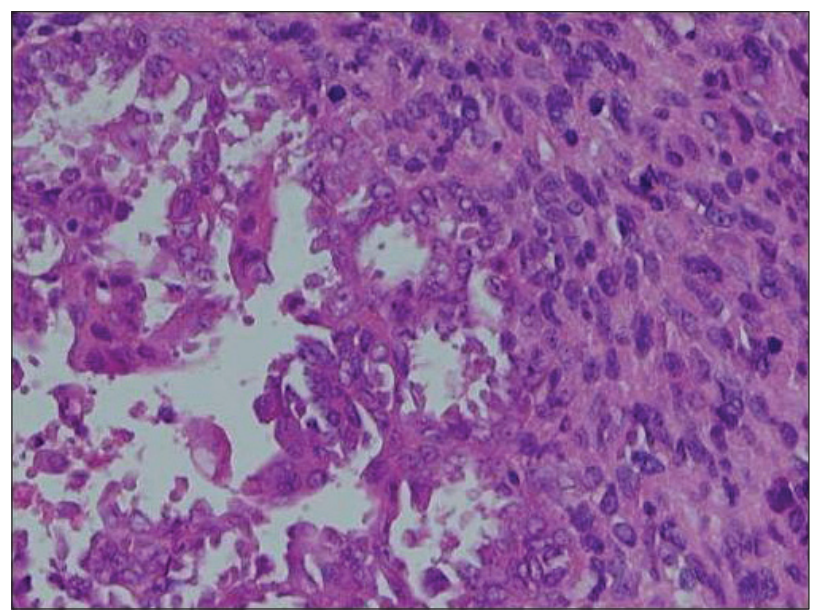

Fig. 4. Biphasic tumor composed of poorly differentiated carcinoma nests (left) and sarcomatous elements (right) $(\mathrm{H} \& \mathrm{E}, \times 200)$. 

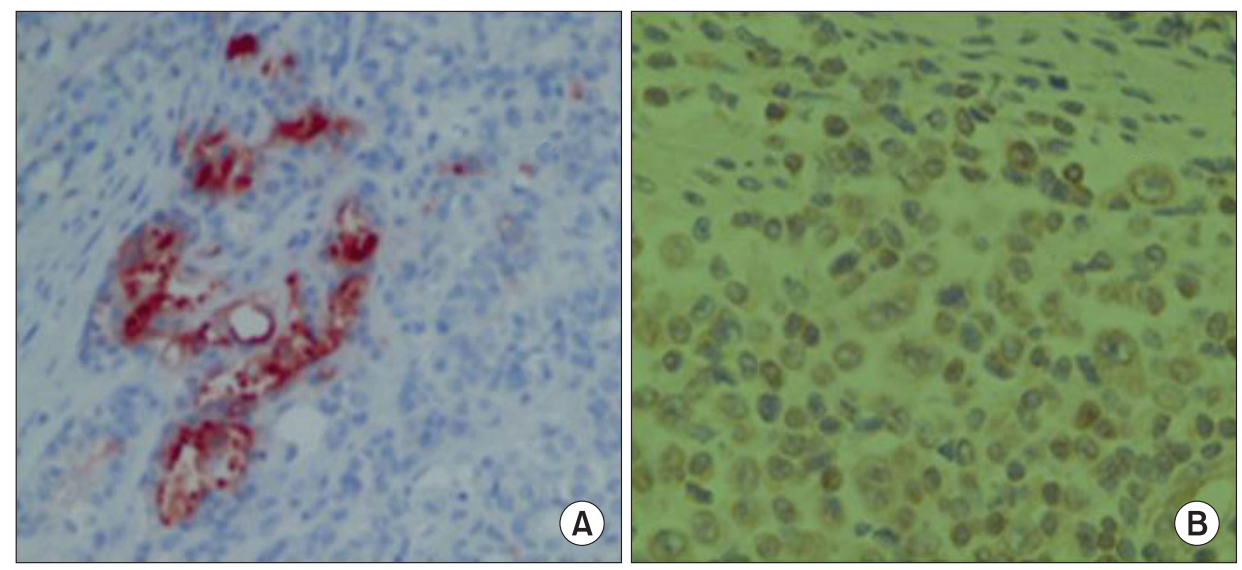

Fig. 5. Carcinoma nests stain $(\times 400)$. (A) Carcinoma nests stain positively for cytokeratin, and (B) sarcomatous elements for vimentin.

enolase). Sarcoma component was posivitve for neurogenic, fibroblastic, smooth muscle acting differentiation.

These IHC findings led to a diagnosis of gastric carcinosarcoma and post-operative course was unremarkable. The patient underwent administration of taxane-based chemotherapy (cisplatin, 5-fluorouracil and docetaxel, administered at 3 week interval). After the patient had received 9th chemotherapy and lost follow up afterwards.

\section{Discussion}

Carcinocarcoma is co-existing biphasic tumor with carcinoma components and sarcoma components. It typically occurs in the uterus, esophagus, lung and thyroid, but rarely in the stomach. ${ }^{4}$ In 1904, Quecken-stadt first reported. About 50 cases of gastric carcinosarcoma have been reported so far, mostly in Japan, which predominantly consist of male population over the age of 60 years. $^{2,4-6}$ In 1991, Kim et al. ${ }^{7}$ first reported the case of carcinosarcoma in the stomach in Korea. This is the only one case report about carcinosarcoma in the stomach, reported in Korea until recently.

Endoscopic finding is generally polypoid, exophytic, or endophytic. Since their surfaces are ulcerated and they frequently infiltrate the gastric wall by forming large tumor masses, they are often mistaken for Borrmann III adenocarcinoma. Endoscopic biopsy of our case revealed malignant round cell neoplasm with poor differentiation, and thus further IHC staining was required for more accurate diagnosis. Clinically, carcinosarcomas do not differ from gastric adenocarcinomas, and discriminating diagnosis is impossible endoscopically or radiologically.

Histological identification of the coexistence of carcinomatous and sarcomatous components has been confirmed by using con- ventional H\&E staining, with the additional use of IHC analyses in some cases. ${ }^{1,3,8}$ Stainings for tumor markers with high sensitivity to carcinomatous components are CEA, EMA, pancreatin, chromogranin A, CD56, synaptophyrin, while desmin, vimentin, $\alpha$-smooth muscle/sarcomeric actin show affinity for the sarcomatous components. ${ }^{1,9}$

Carcinosarcoma is classified into three types based on its origin. In type I, 'collision' tumors, there is a distinct boundary between the two tumor components. In type 2, or 'combinded' tumors, the two tumor components are overlapping, though the stromal component does not have any distinguishing characteristics. In type 3 , or 'composite' tumors, both two tumor types are present with different characteristics of stromal components. ${ }^{1,10}$ In our case was confirmed type 1 group with the use of IHC staining.

Radical gastrectomy is regarded as the only curative treatment for gastric carcinosarcoma. However, this tumor was in advanced stage at diagnosis and had worse prognosis than other types of gastric carcinoma even with radical gastrectomy. ${ }^{7}$ The mean survival period is estimated to be 10 15 months, while greater than $50 \%$ of tumor recurrence occurs in the first postoperative year mainly in peritoneum. ${ }^{89}$ As gastric carcinosarcoma is very rare, the effects of chemotherapy or radiotherapy have not yet been reported. He et $\mathrm{al}^{11}$ reported possible treatment of tumor reduction with methionine/valine-depleted enteral nutrition, although its efficacy in humans is ambiguous and remains to be established. Resulted tumor growth suppression in rat was because of protein and nucleic acid synthesis inhibition by methionine/valine-depleted enteral nutrition.

In conclusion, carcinosarcoma in stomach is rare tumor with high malignant potential, it tends to have poor prognosis. Thus gastric carcinosarcoma should be taken into consideration as a part of differential diagnosis when a gaint tumor is not exactly diag- 
Choi KW, et al.

nosed, and IHC stain is a critical method in making an accurate diagnosis of carcinosarcoma, in case of we cannot determined specific histologic type of the tumor by conventional H\&E stain because of some reasons, for example severe tissue necrosis and hemorrhage.

\section{References}

1. Bansal M, Kaneko M, Gordon RE. Carcinosarcoma and separate carcinoid tumor of the stomach. A case report with light and electron microscopic studies. Cancer 1982;50:1876-1881.

2. Tsuneyama K, Sasaki M, Sabit A, Yokoi K, Arano Y, Imai T, et al. A case report of gastric carcinosarcoma with rhabdomyosarcomatous and neuroendocrinal differentiation. Pathol Res Pract 1999;195:93-97.

3. Kitamura S. Study on carcinosarcoma of stomach. Gann 1950;41:15-26.

4. Tanimura H, Furuta M. Carcinosarcoma of the stomach. Am J Surg 1967;113:702-709.
5. Quekenstadt H, ed. Uber Karzinosarkome Leipzig, 1904.

6. Kayaselcuk F, Tuncer I, Toyganözü Y, Bal N, Ozgür G. Carcinosarcoma of the stomach. Pathol Oncol Res 2002;8:275-277.

7. Kim BB, Choi WJ, Park HR. Carcinosarcoma of the stomach. J Korean Surg Soc 1991;40:113-120.

8. Teramachi K, Kanomata N, Hasebe T, Ishii G, Sugito M, Ochiai A. Carcinosarcoma (pure endocrine cell carcinoma with sarcoma components) of the stomach. Pathol Int 2003;53:552-556.

9. Kawabata Y, Nakai T, Ooba K, Okumura T, Matsuura Y, Miyazaki $Y$, et al. A case report of carcinosarcoma of the stomach (in Japanese). Jpn J Gastroenterol Surg 1993;26:2189-2193.

10. Sato Y, Shimozono T, Kawano S, Toyoda K, Onoe K, Asada Y, et al. Gastric carcinosarcoma, coexistence of adenosquamous carcinoma and rhabdomyosarcoma: a case report. Histopathology 2001;39:543-544.

11. He YC, Cao J, Chen JW, Pan DY, Zhou YK. Influence of methionine/valine-depleted enteral nutrition on nucleic acid and protein metabolism in tumor-bearing rats. World J Gastroenterol 2003;9:771-774. 